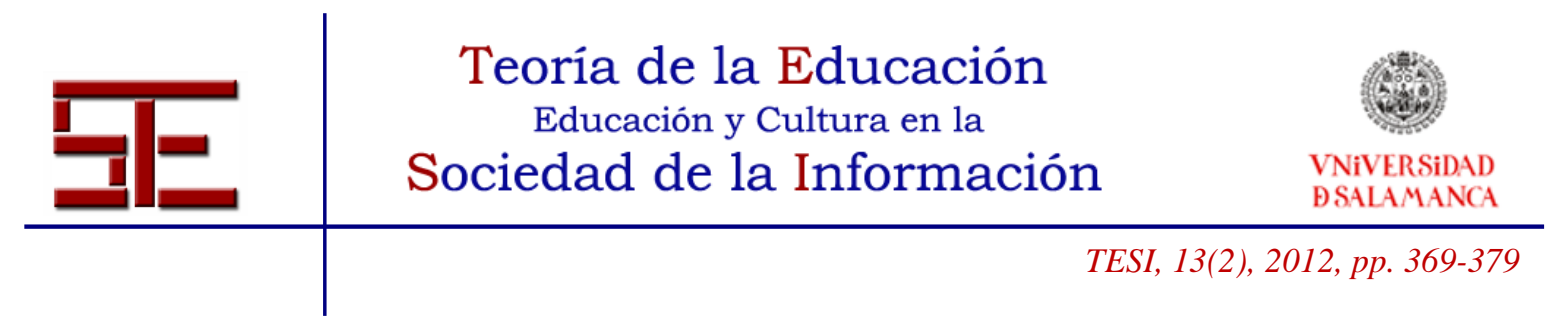

\title{
LA EDUCACIÓN PARA LA AUTONOMÍA MORAL EN LA ESCUELA INTERCULTURAL ${ }^{1}$
}

Resumen: Esta artículo pretende ofrecer elementos para la reflexión en torno a la necesidad de la educación para la autonomía moral en la escuela y los principios pedagógicos susceptibles a tener en cuenta desde la perspectiva intercultural. Para ello se realiza un posicionamiento teórico y conceptual en torno a la educación para la autonomía moral y la ética como referente de la misma, para adentrarnos en la realidad de la escuela multicultural y el reto que implica esta educación para la autonomía moral en un contexto diverso culturalmente, lo cual supone uno de los grandes desafíos en el desarrollo de la educación intercultural en los centros educativos y un mecanismo para el desarrollo de competencias cívicas interculturales, así como para la prevención, gestión y resolución de los conflictos dentro de la comunidad educativa.

Palabras clave: autonomía moral; ética; educación intercultural; multiculturalidad; conflicto.

\footnotetext{
${ }^{1}$ Este artículo se ha desarrollado en el marco del proyecto I+D con código EDU 2009-09654 "De la extranjería a la ciudadanía”. Grupo de Investigación HUM-169 de la Junta de Andalucía.
}

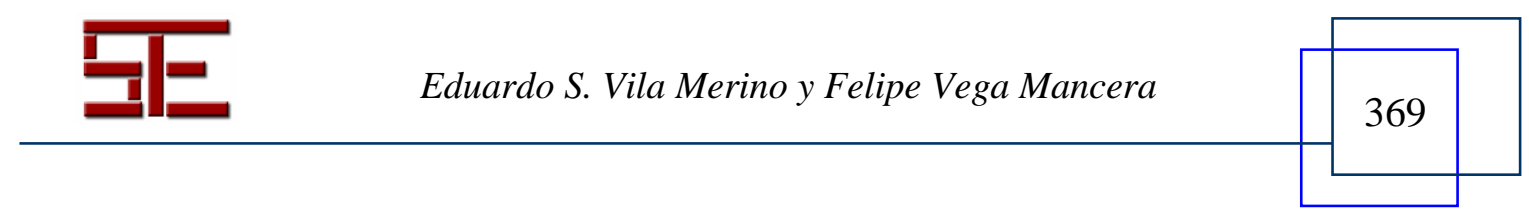




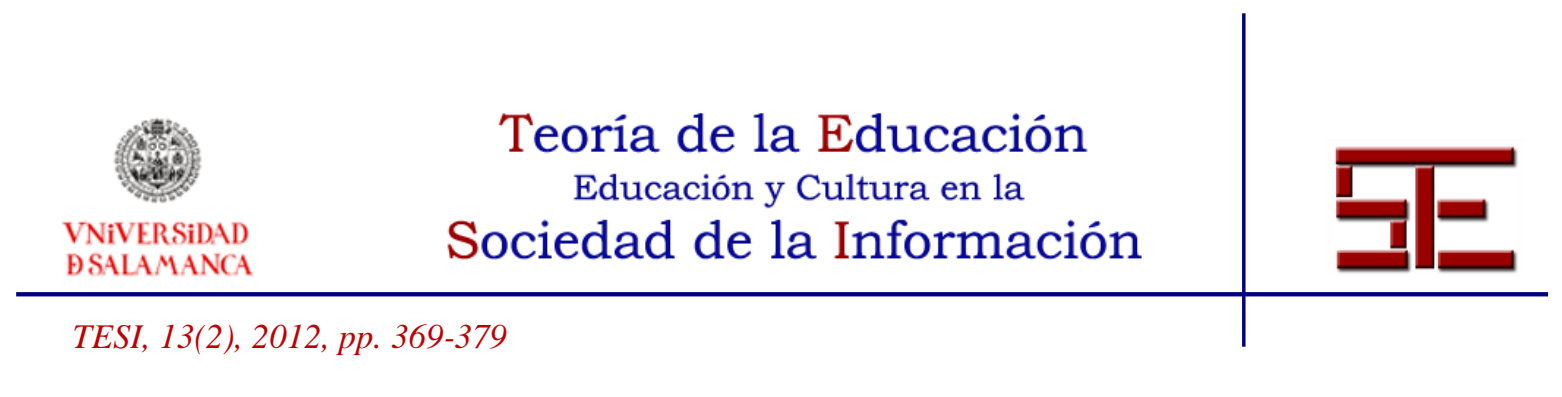

\title{
THE EDUCATION FOR THE MORAL AUTONOMY IN THE INTERCULTURAL SCHOOL
}

\begin{abstract}
This paper tries to offer elements for the reflection concerning the need of the education for the moral autonomy in the school and the pedagogic capable beginning to bearing in mind from the intercultural perspective. For it there is realized a theoretical and conceptual positioning concerning the education for the moral autonomy and the ethics as modal of the same one, to enter in the reality of the multicultural school and the challenge that involves this education for the moral autonomy in a culturally diverse context, which supposes one of the big challenges in the development of the intercultural education in the educational centers and a mechanism for the development of civic intercultural competences, as well as for the prevention, management and resolution of the conflicts inside the educative community.
\end{abstract}

Keywords: moral autonomy; ethics; intercultural education; multiculturalidad; conflict.

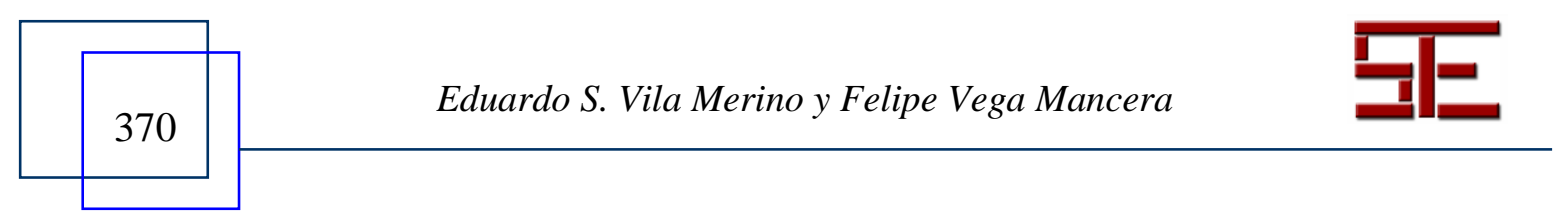




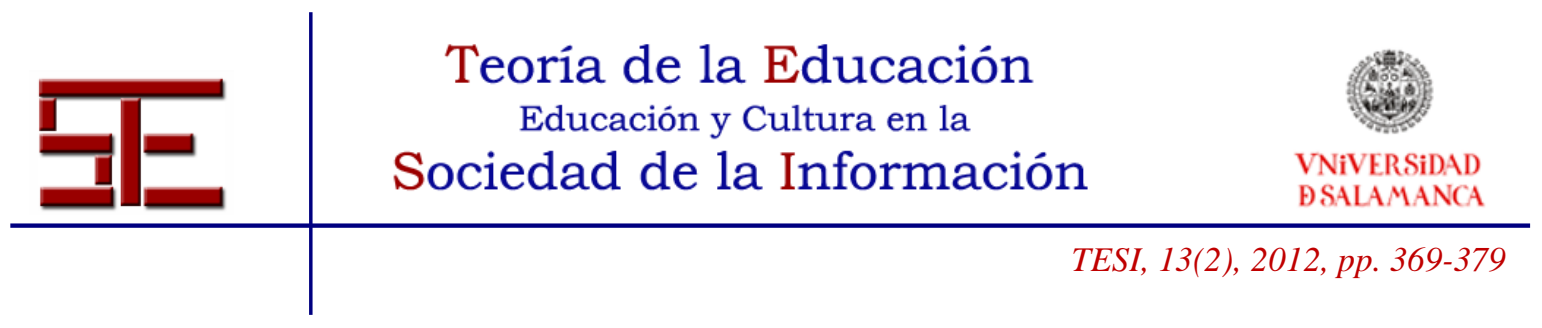

\title{
LA EDUCACIÓN PARA LA AUTONOMÍA MORAL EN LA ESCUELA INTERCULTURAL
}

Fecha de recepción: 20/09/2011; fecha de aceptación: 20/10/2011; fecha de publicación: 26/07/2012

\author{
Eduardo S. Vila Merino \\ eduardo@uma.es \\ Universidad de Málaga \\ Felipe Vega Mancera \\ felipevega@uma.es \\ Universidad de Málaga
}

\section{LA RELACIÓN EDUCATIVA Y LA AUTONOMÍA MORAL}

La relación educativa es uno de los puntos clave de la teoría de la educación. De hecho, se torna en el epicentro de muchas de las más brillantes reflexiones pedagógicas, desde Freire a Esteve. Este último nos decía que "se trata de una relación que tiene en sí una finalidad que de alguna manera tiene como objeto al educando; pero que tiene que contar a cada paso con la condición de sujeto que le corresponde como persona. Corremos en cada instante el riesgo de olvidar al sujeto y hacerlo un mero objeto de condicionamiento; y al mismo tiempo, en la vertiente opuesta, el riesgo de olvidar la construcción de la personalidad, haciendo banal la relación, y olvidando dirigirla hacia una finalidad intencional elegida" (Esteve, 2008, 2-3). Esto implica que nos movemos siempre, como educadoras y educadores, en un difícil equilibrio entre el dogma y la libertad de pensamiento, entre la autonomía y la sumisión, entre la creatividad y el mecanicismo en el quehacer educativo.

Al hilo de lo anterior, uno de los aspectos centrales de estas disyuntivas pedagógicas tiene que ver con el desarrollo de la autonomía del educando, y específicamente con el desarrollo de la autonomía moral, entendida como capacidad de tomar decisiones, de discernir desde nuestra propia responsabilidad personal para que nuestras reflexiones y acciones se den en un marco intencional y con fines, al mismo tiempo que teniendo en cuenta las consecuencias de las mismas, dotándolas así de sentido. Para desarrollo de la misma, coincidimos de nuevo con Esteve $(2010,130)$ al señalar que: "Una de las peores patologías de la relación educativa consiste en querer prolongar la situación de dependencia. Educamos para la autonomía. Educamos para que nuestros hijos y nuestros alumnos sean capaces, un día, de seguir su propio camino. (...) Reconocer su

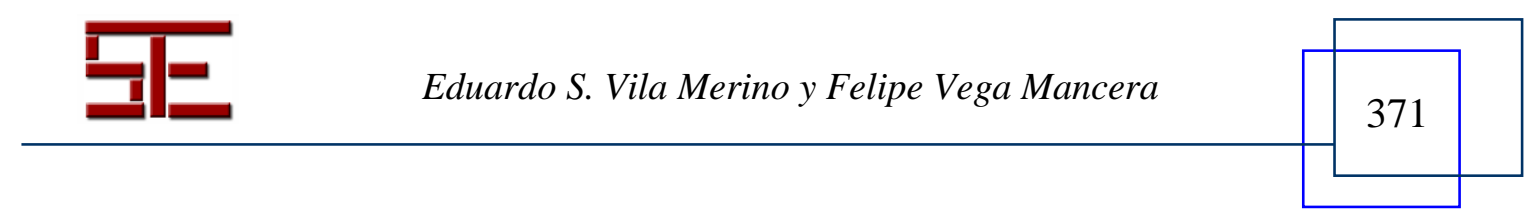




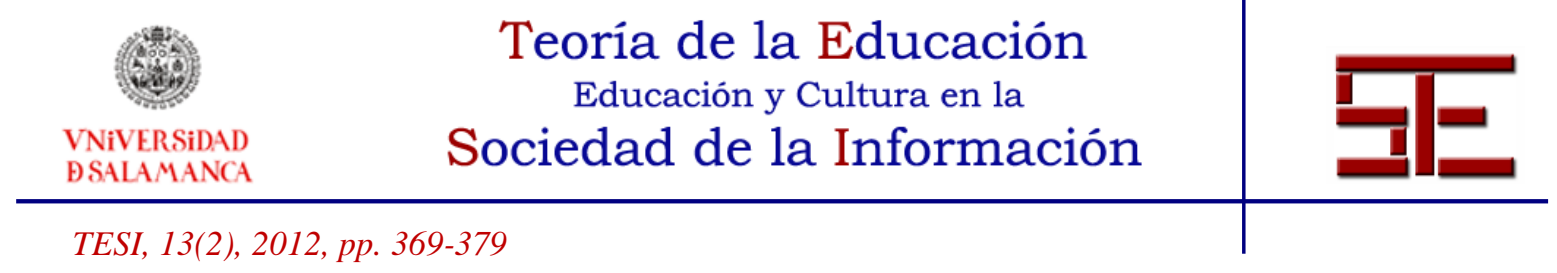

autonomía y aceptar que tomen sus propias decisiones es la prueba definitiva de que hemos tenido éxito en la relación educativa" (Esteve, 2010,130).

Por tanto, la relación educativa desde la óptica de la de los derechos y la autonomía del educando se encuentra basada en unos principios donde la confianza en las competencias del otro u otra, las expectativas positivas en sus logros, resulta fundamental, pues las mismas condicionan las respuestas que obtenemos de los demás. $\mathrm{Y}$ es que los espacios relacionales de calidad se construyen a partir de la calidad de las relaciones que los configuran. De ahí que la generación de espacios democráticos que primen la construcción identitaria en libertad y la educación para la autonomía moral sea una cuestión de ineludible asunción en la reflexión teórica de la educación, ya que "el práctico tiene que tomar decisiones y elaborar juicios reflexionantes, precisamente porque hay incertidumbre, porque nada está dado de antemano, porque tiene que atravesar, por sí mismo, la praxis que realiza, y porque tiene que aprender en qué consisten sus artes" (Bárcena, 2005, 184).

Claro está que todo lo anterior debe ser contextualizado para que cobre un sentido de autenticidad, por lo que no podemos hacer los análisis de forma ajena al contexto socioeconómico en el que estamos insertos, así como a la pluralidad cultural que conforma los espacios sociales, incluida la escuela. En este sentido, educar para la autonomía moral en contextos multiculturales implica tener presente la variable transversal que supone la interculturalidad como concepto y herramienta imprescindible en la tarea educativa, máxime en sociedades complejas como las nuestras, donde se aúna tanto como se contrapone lo híbrido con lo hegemónico, el pensamiento único con la diversidad, el relativismo con lo contingente. Por ello debemos tener presentes planteamientos como los siguientes:

"Los analistas sociales coinciden en señalar que nos hallamos en una situación contradictoria; de un lado, la adquisición temprana de valores centrados en la autonomía cultural y moral, y de otro lado, la cada vez mayor dependencia de los jóvenes de su red familiar, al resultarles más difícil lograr la autonomía material. El deseo de autonomía de los adolescentes y jóvenes contrasta, por otra parte, con la fragilidad que muestran a la hora de asumir responsabilidades y tomar decisiones en diferentes ámbitos de su vida" (Romero, Bernal y Jiménez, 2009, 109).

Hay autores y autoras que nos hablan también de una paulatina desaparición de la niñez y una prolongación de la adolescencia, lo cual puede entrar en conflicto también con diferentes concepciones de las mismas que se pueden encontrar entre diversos grupos de culturales de referencia en relación en las aulas. Por eso estas cuestiones también

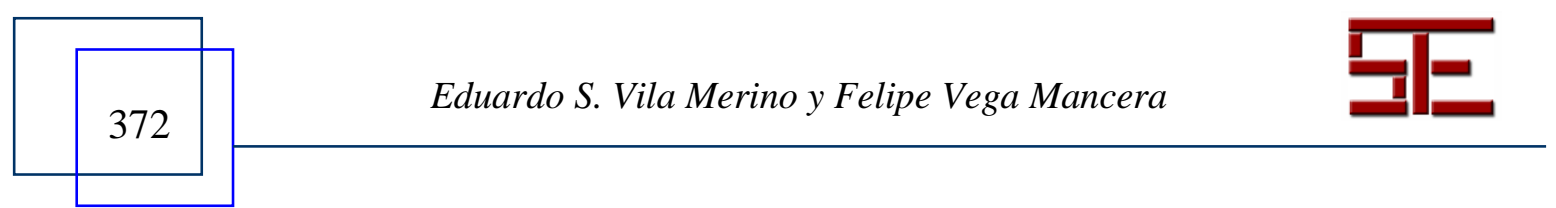




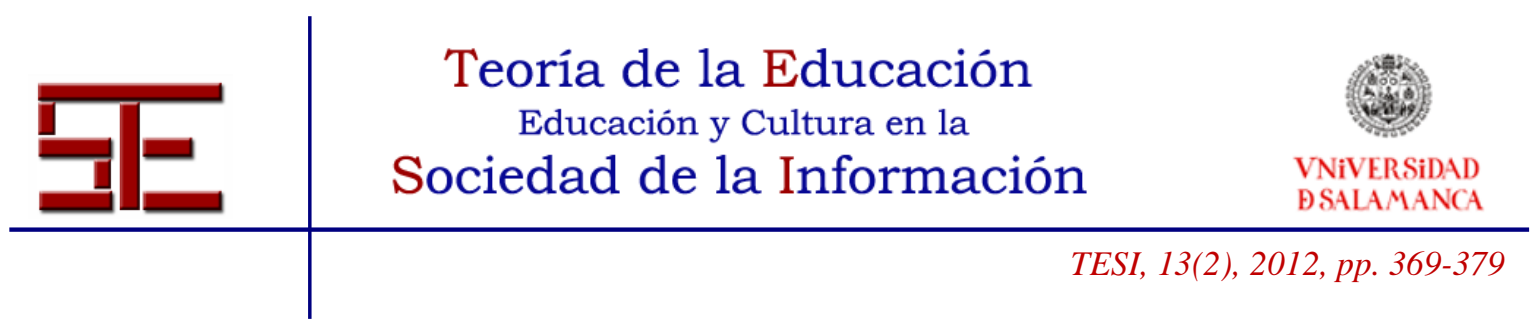

debemos tenerlas en cuenta a la hora de plantear la educación para la autonomía moral y resulta importante realizar reflexiones donde se valore lo social y lo cultural como aspectos que dan lugar a situaciones que deben ser asumidas en el discurso teórico y constituir fuentes desde las que emerja la praxis educativa intercultural.

\section{LA IDENTIDAD Y LA EDUCACIÓN PARA LA AUTONOMÍA MORAL EN CONTEXTOS MULTICULTURALES}

La configuración de la identidad y el desarrollo de la autonomía moral se encuentran estrechamente relacionadas, en la medida, entre otras cuestiones, en que la capacidad de discernimiento que la segunda implica ayuda a construirnos identitariamente dentro de la multiplicidad de culturas en interacción que configuran nuestro camino vital. Por eso es necesario tener presente la encrucijada que a menudo supone esa interacción y cómo condiciona nuestras decisiones. Para ello resulta interesante partir de dos premisas, cuales son las de evitar la proyección de un determinado modelo cultural como algo universal y la de asumir que toda acción educativa supone, de algún modo, una forma de aculturación. Desde esta perspectiva, cierto es que la tendencia social, fomentada desde los modelos de escuela segregadores, suele conllevar la consideración de la cultura hegemónica como algo monolítico y 'natural' a lo que hay que 'integrarse', lo cual provoca tantas adhesiones como conflictos. Esto nos lleva a situaciones en las que las personas tienen que ajustarse a una cultura que es significativamente diferente a la suya, por lo cual a menudo se sienten confundidos, extrañados, ansiosos y frustrados, porque desconocen cuáles son sus expectativas en las diferentes situaciones. Con frecuencia no pueden solucionar problemas interpersonales y no conocen las conductas que son aceptadas o no por la nueva cultura. En este sentido, nos encontramos con que: "Muchos chicos y chicas hijos/as de inmigrantes experimentan conflictos de identidad durante los procesos de aculturación porque por una parte se sienten presionados por su familia y comunidad para mantener los valores tradicionales de su cultura, y por otra, se sienten presionados por la escuela y la sociedad de acogida para que aceptan unos nuevos valores" (Soriano, 2004, 186). Y esto tiene consecuencias importantes de cara al proceso educativo. En todo caso, siguiendo con la misma autora, debemos tener presente que: "Los compromisos culturales se distribuyen de manera desigual: unas personas le dan más importancia que otras al grupo de pertenencia, no todas participan por igual de una cultura, ni coincide en intensidad con la que se identifica con su grupo. (...) Por eso nos surge un interrogante cuando personas de diferentes grupos culturales 'conviven' juntas, ¿con qué grupo se identifican?” (Soriano, 2004, 187).

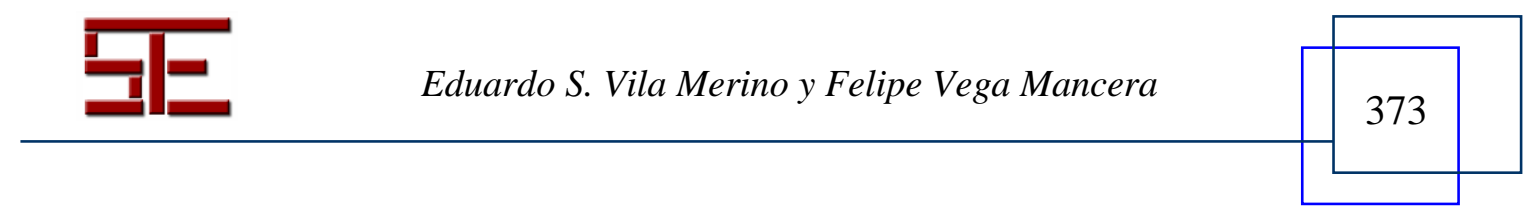




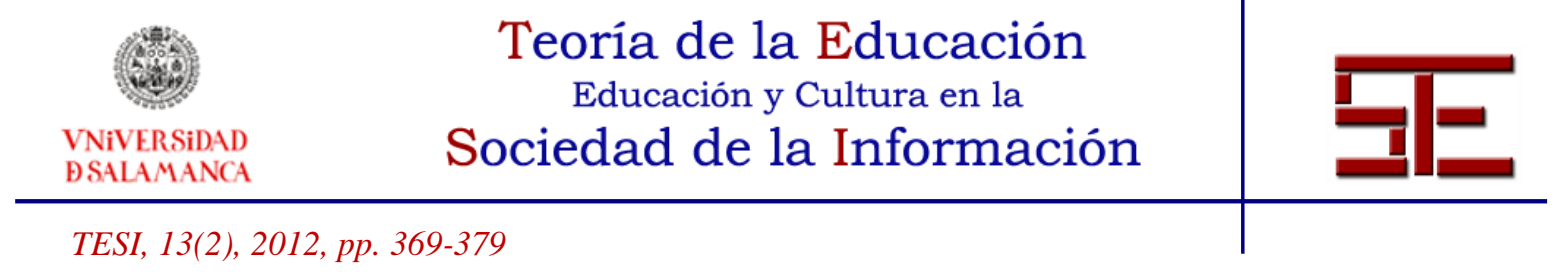

En esta segunda cuestión podemos entrever la impronta de la autonomía moral de las personas como un factor determinante también a la hora de la asunción acrítica o no de los valores culturalmente transmitidos, lo que la pone más en valor si cabe. Desde esta perspectiva, lo intercultural en la construcción identitaria alude a una dialéctica entre lo colectivo y lo individual que conlleva, por un lado, la reconstrucción de las identidades particulares ligada a la compleja pluralidad social existente y, por otro, la necesidad de contar con una nueva forma de identidad colectiva de índole política generada a partir de las anteriores y que las trascienda, evitando asimetrías per se entre las distintas culturas, introduciendo criterios de justicia como eje de la construcción de un común transversal entre las mismas y posibilitando confluencias en los espacios públicos compartidos como concreción del 'espacio moral' entre culturas que supone la interculturalidad desde mi 'universalismo' sostenido no contra las diferencias culturales ni al margen de ellas, sino desde y a través de ellas (Pérez Tapias, 2007).

Retomando entonces esta vertiente política en la reflexión moral y la construcción identitaria con vocación intercultural, resulta interesante partir de la idea de Arendt en torno al carácter representativo del pensamiento político con un potencial pedagógico intercultural claro:

"El pensamiento político es representativo; me formo una opinión tras considerar determinado tema desde diversos puntos de vista, recordando los criterios de los que están ausentes; es decir, los represento. Este proceso de representación no implica adoptar ciegamente los puntos de vista reales de los que sustentan otros criterios y, por tanto, miran hacia el mundo desde una perspectiva diferente; no se trata de empatía, como si yo intentara ser o sentir como alguna otra persona, ni de contar cabezas y unirse a la mayoría, sino de ser y pensar dentro de mi propia identidad tal y como en realidad soy. Cuantos más puntos de vista diversos tenga yo presentes cuando estoy valorando determinado asunto, y cuando mejor pueda imaginarme cómo sentiría y pensaría si estuviera en lugar de otros, tanto más fuerte será mi capacidad de pensamiento representativo y más válidas mis conclusiones, mi opinión" (Arendt, cit. Bárcena, 2005, 187).

Educativamente, esa polifonía necesaria para el buen juicio, siguiendo términos aristotélicos, vinculado al desarrollo de la autonomía moral, debe tener un reflejo en tres cuestiones básicas:

La asunción de la diversidad de pertenencias que configura nuestra identidad.

"Especialmente en el caso de todas las personas cuya cultura de origen no coincide con la cultura de la sociedad en que viven, es necesario que puedan asumir, sin demasiados desgarros, esa doble pertenencia, que puedan mantener su apego a su cultura de origen, no sentirse obligados a disimularla como si fuera

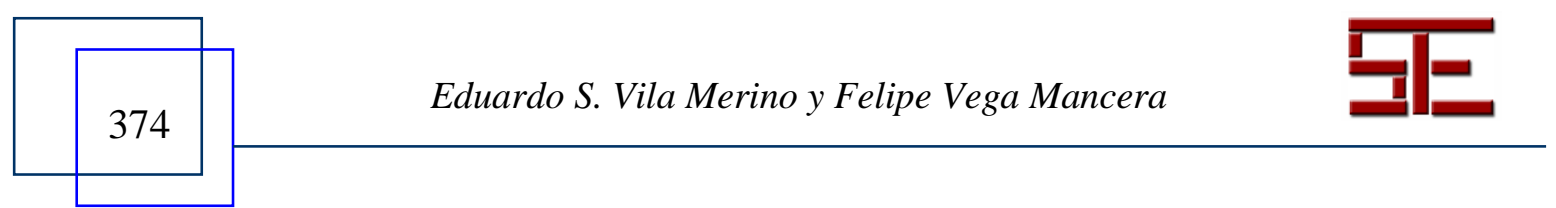




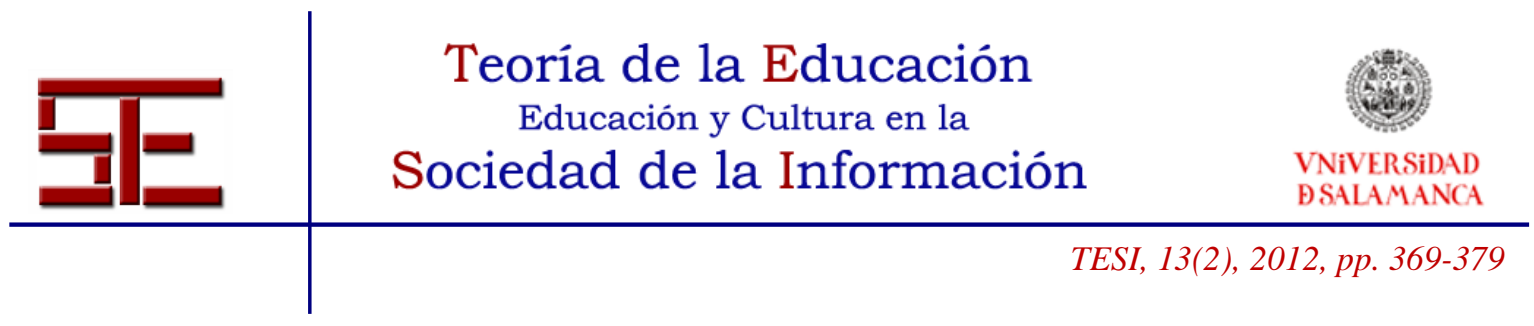

una enfermedad vergonzante y abrirse en paralelo a la cultura del país de acogida" (Maalouf, 1999, 207).

El carácter normativo de la educación y la necesidad por tanto de una permanente reflexión ética en ese quehacer, teniendo claro que:

En todas nuestras interacciones con los niños, estamos involucrados, tanto si nos gusta como si no, en distinguir lo que es bueno para ellos de lo que no lo es (Van Manen, 1998, 16).

La cuestión de los límites, a través de los cuales se genera la propia libertad de expresión, de pensamiento, etc., lo cual se encuentra en el epicentro del proceso de desarrollo de la autonomía moral, en la línea que plantea Freire (2003, 39):

Es preciso defender el derecho que tiene el niño de preguntar, de satisfacer su curiosidad, pero al mismo tiempo decirle que hay momentos para preguntar y momentos para abstenerse, lo que en ética definimos 'asumir los límites de la libertad'. Sin límites no hay libertad, como tampoco hay autoridad. La formidable cuestión que se nos plantea a los educadores es cómo establecer los límites, en qué consisten realmente, y cuáles entre todos ellos son los que hay que implementar.

\section{LA AUTONOMÍA MORAL EN LA ESCUELA INTERCULTURAL}

Todo lo anterior, inscrito en un contexto multicultural sin precedentes, nos debe hacer aterrizar en la escuela como institución educativa, teniendo en cuenta que "más que los 'inmigrantes' o los 'diferentes', el objeto de la pedagogía intercultural debe ser las propias instituciones educativas, los espacios de educación y los discursos pedagógicos en tanto que configuradores de unas formas concretas de identidad" (Marí Ytarte, 2007, 25).

Entonces la pregunta clave sería por qué y cómo trabajar la educación para la autonomía moral en contextos escolares de tanta diversidad cultural y con valores morales en conflicto desde la interacción, asumiendo un concepto de conflicto como algo necesario para el avance social y educativo. Desde la teoría de la educación consideramos fundamental para esto tener presentes los siguientes planteamientos de partida:

a) Las personas somos agentes activos de cultura cuya identidad va pareja a la complejidad de las formas sociales e intercambios individuales y colectivos en los que nos vemos envueltos. La riqueza de los mismos por tanto redundará en una construcción

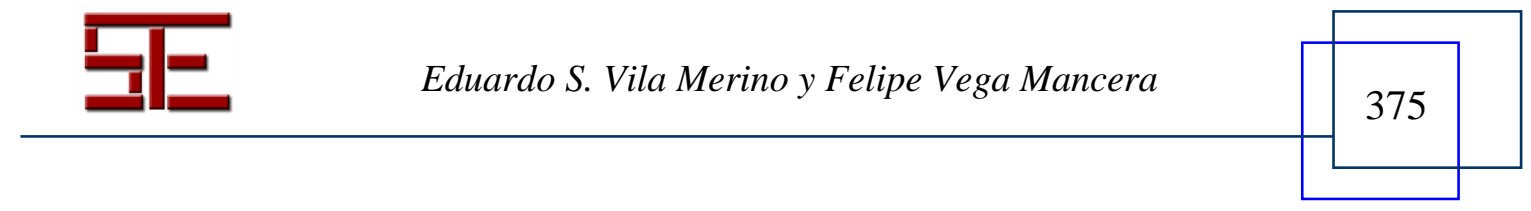




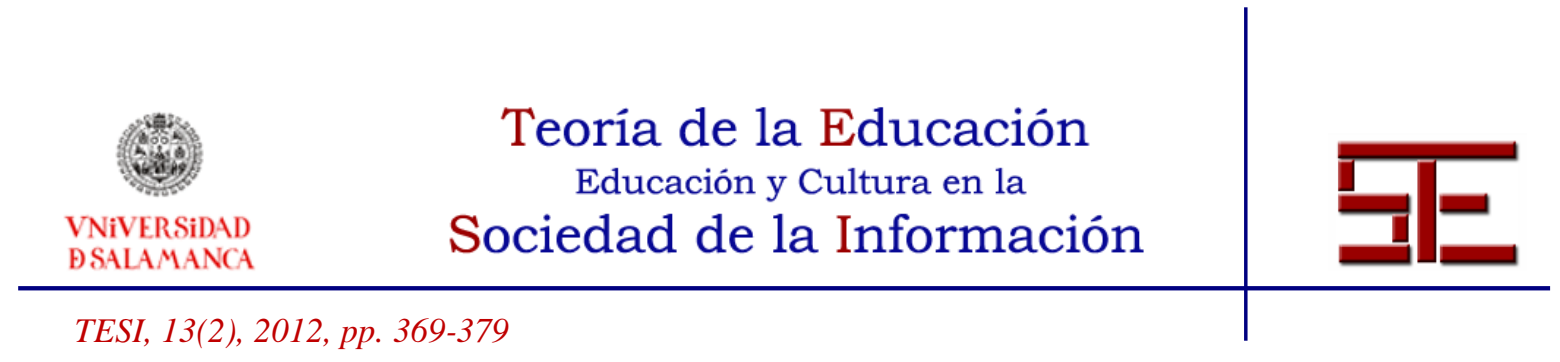

identitaria con menos posibilidades de ser encorsetada desde lo hegemónico o desde posiciones de privilegio arbitrarias de unas culturas sobre otras.

b) Es necesario no reducir al estereotipo o al 'folklore' las culturas ni condicionar nuestras relaciones con el otro/a cultural a su adscripción más visible, ya que su grado de identificación personal con su cultura de origen o referencia puede ser muy variable $\mathrm{y}$, en todo caso, no resulta justo ni realista circunscribir en base a prejuicios culturales las concepciones y comportamientos de los individuos ni realizar predicciones sobre los mismos.

c) La noción de traducción entre culturas es aquí fundamental, en la medida en que supone un esfuerzo para conocer y comprender (que no necesariamente compartir acríticamente) cuestiones morales para su valoración, siendo el papel de la interculturalidad un referente ineludible en este sentido, de manera que los 'significados' de partida para la traducción intercultural constituyen un desafío profundamente ligado a las propias diferencias vinculadas a la construcción de la autonomía moral de las personas, teniendo en cuenta incluso no ya la dificultad de los mecanismos de traducción cultural en sí, sino la propia asunción de la intraductibilidad de determinados enunciados culturales, otorgándosele ahí a la interculturalidad una dimensión mayor en la necesidad de ejercer como instrumento para tender puentes de mínimos morales para el encuentro.

d) En coherencia con lo anterior, "pensar la educación como apertura de las identidades, y no tanto como su progresiva adecuación a un modelo de cultura o a un modelo de sociedad identitariamente definido. Desde este lugar, la intercultural no sería un objetivo de la educación, no configuraría una meta deseable de la convivencia, sino su punto de partida incondicional. Más que como tarea a largo plazo, más que como resolución de los conflictos y las problemáticas de la diversidad, la intercultura sería la materia prima de la acción educativa, su espacio de realización” (Marí Ytarte, 2007, 121).

Finalmente, partiendo de los principios anteriores y relacionándolos con la educación para la autonomía mora, vamos a desarrollar una serie de puntos a modo de prioridades para su consecución en el marco específico de la construcción de una escuela intercultural (basado en Vila Merino, 2012):

- Identificar las representaciones de la alteridad presente y ausente que todos y todas construimos, de manera que los procesos educativos morales favorezcan la concientización, que diría Freire, de esas representaciones del otro o la otra diferente, las cuales nos hacen situarnos en nuestro contexto concreto y nuestra forma de ver el mundo desde nuestras percepciones y comprensión de la diversidad.

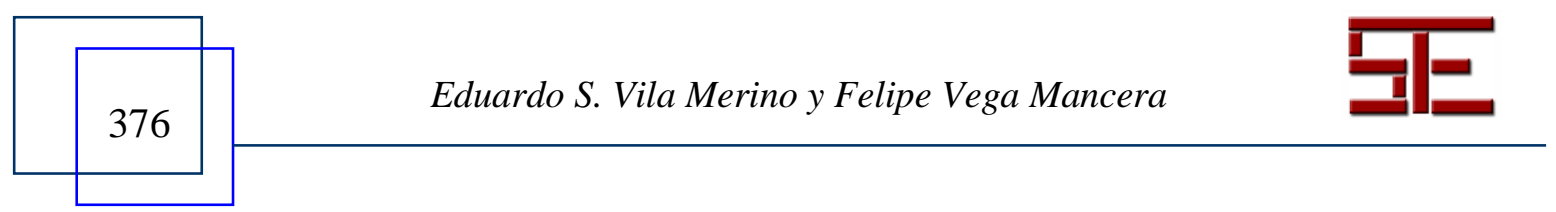




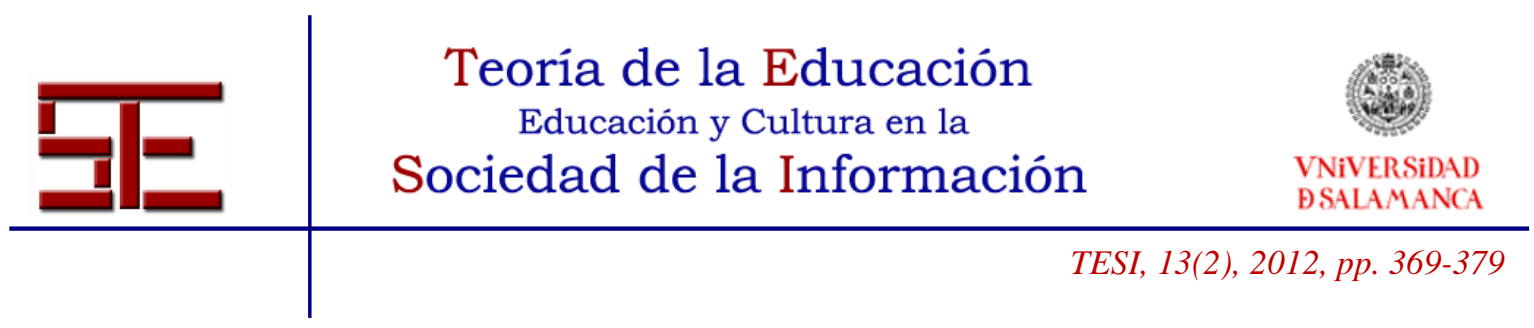

- Profundizar en la configuración de los prejuicios y discriminaciones presentes en nuestras sociedades, evitando su naturalización y reivindicando la lucha contra los mismos en tanto que simplificaciones segregadoras de la realidad con fuertes cargas cognitivas, culturales y emocionales a las que desde la educación para la autonomía moral debe darse respuesta a través del análisis crítico de situaciones y hechos concretos desde una perspectiva dialógica.

- Cuestionar el statu quo de ciudadanía basada en una perspectiva monocultural o etnocéntrica. Esto nos debe llevar a la visibilización de la pluralidad cultural para abrir espacios de construcción de nuevos desarrollos de la ciudadanía desde el referente de la interculturalidad, así como reconocer nuestra propia identidad cultural desde una óptica inclusiva y siendo conscientes de que vivimos permanentemente en un cruce de culturas desde el que reinventamos nuestras identidades.

- Visualizar la alteridad presente en nosotros y nosotras, es decir, ser capaces de mirarnos siendo conscientes de que lo que somos y nuestra historia lo es desde la interrelación con la de los y las demás, por lo que la educación para la autonomía moral debe ayudarnos a observarnos a nosotros y a nuestras relaciones de otra manera, más plural y dialéctica, y un buen instrumento para ello son las historias de vida concretas, los dilemas morales de contenido intercultural o las aportaciones de los medios de comunicación y las TIC en este sentido.

- Conjugar la promoción de políticas de igualdad (equidad) y políticas de identidad (reconocimiento) tanto a nivel macro como en la micropolítica de la acción educativa, fomentando así una ciudadanía activa, el empoderamiento de las culturas no hegemónicas y unas prácticas educativas sustantivas vinculadas con la propia praxis de los derechos humanos de primera, segunda y tercera generación.

- Buscar la cooperación entre los diversos ámbitos educativos, formales y no formales, en beneficio de una educación pública de calidad que dé respuestas a los desafíos cívicos de nuestras sociedades multiculturales. Para ello no son suficientes didácticamente momentos puntuales o específicos de tratamiento más o menos artificial de las alteridades culturales, sino que se requiere además de la promoción de experiencias de diálogo en profundidad con el otro/a cultural y de asunción de su papel social igualitario desde las diferencias que nos legitiman para la convivencia.

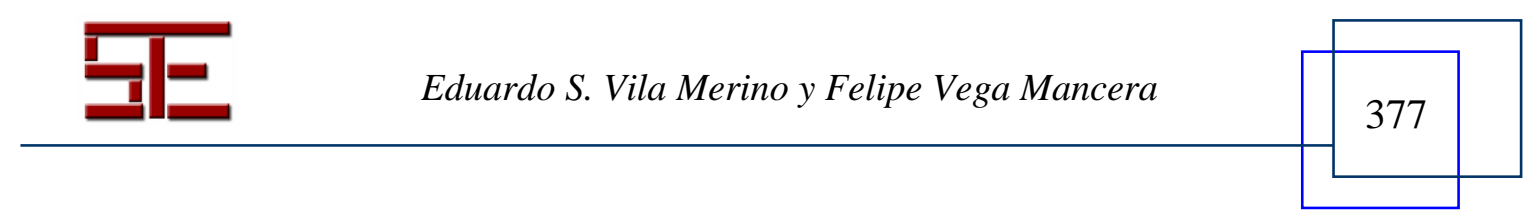




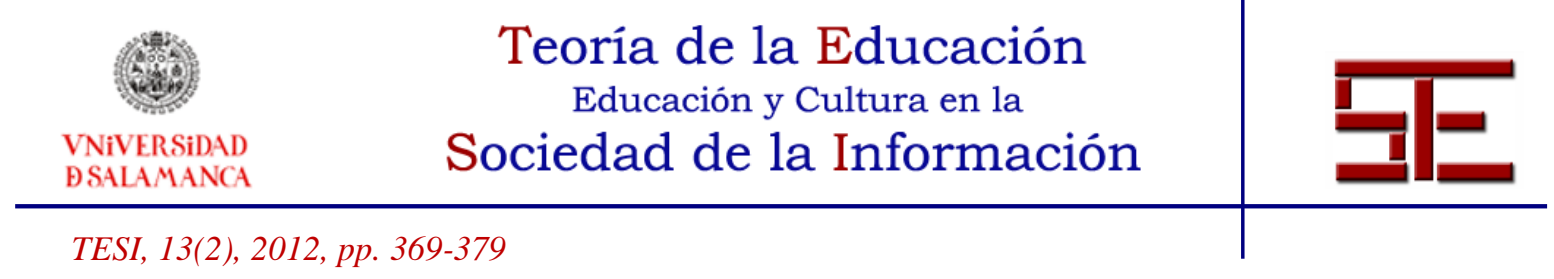

Pensar la escuela intercultural es por tanto también promover la autonomía moral de las personas como categoría necesaria de cara a la formación de una ciudadanía crítica que apuesta por la convivencia y la justicia social para todas y todos.

\section{4.- BIBLIOGRAFÍA}

Esteve, J.M. (2008). La urdimbre de la relación educativa. Addenda presentada al XXVIII Seminario Interuniveristario de Teoría de la Educación. Universidad de Oviedo.

- (2010). Educar: un compromiso con la memoria. Un libro para educar en libertad. Barcelona, Octaedro.

Freire, P. (2003). El grito manso. Siglo XXI, Buenos Aires.

Maalouf, A. (1999). Identidades asesinas. Alianza, Madrid.

Marí Ytarte, R. Ma (2007). ¿Culturas contra ciudadanía? Gedisa, Barcelona.

Pérez Tapias, J. A. (2007). Del bienestar a la justicia. Aportaciones para una ciudadanía intercultural. Trotta, Madrid.

Romero, C., Bernal, A. y Jiménez, J.R. (2009): Tejiendo vínculos: la textura de la relación educativa. En Peña, J. V. y Fernández, C. M (Coords.): La escuela en crisis. Octaedro, Barcelona.

Soriano, E. (2004). Identidad y ciudadanía: dos retos de la práctica educativa intercultural. En Soriano, E. (coord.): La práctica educativa intercultural. La Muralla, Madrid.

Van Manen, M. (1998). El tacto en la enseñanza. Paidós, Barcelona.

Vila Merino, E. S. (2012). Un juego de espejos: pensar la diferencia desde la pedagogía intercultural. Revista Educación XXI, Volumen 15,2.

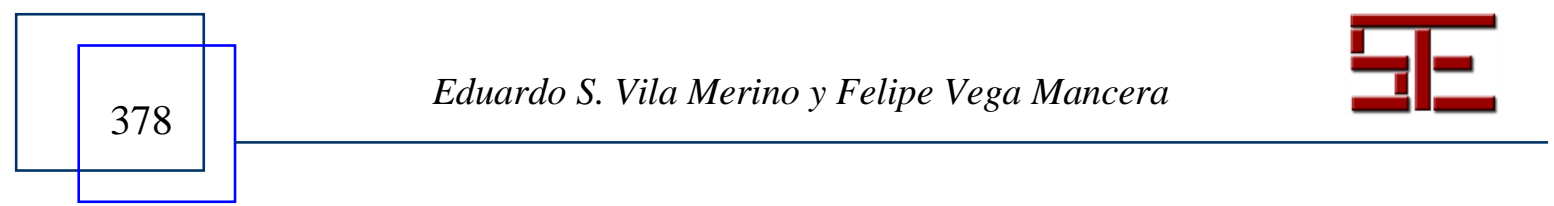




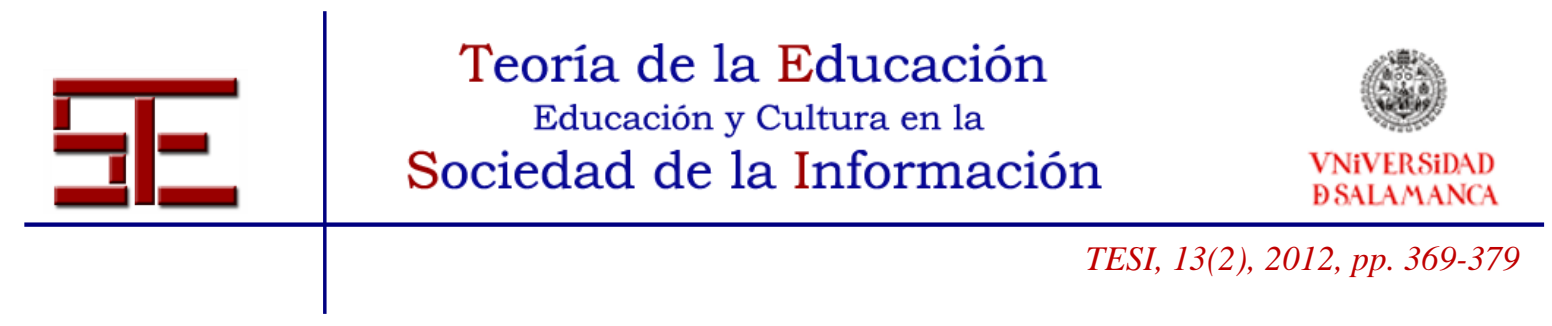

Para citar el presente artículo puede utilizar la siguiente referencia:

Vila Merino, E. S. y Vega Mancera, F. (2012). La educación para la autonomía moral en la escuela intercultural. Revista Teoría de la Educación: Educación y Cultura en la Sociedad de la Información. 13(2), 369-378 [Fecha de consulta: dd/mm/aaaa].

http://campus.usal.es/ revistas_trabajo/index.php/revistatesi/article/view/9013/9257

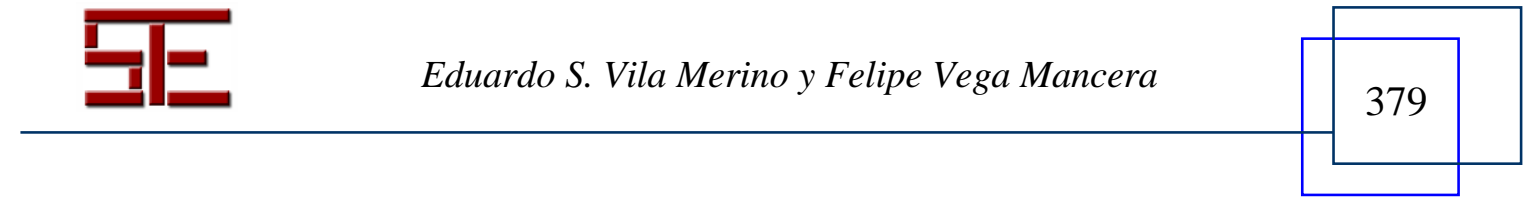

